\title{
Robots in a condensed matter lab
}

\section{Automated tuning of inter-dot tunnel couplings in quantum dot arrays} Authors: C. J. van Diepen, P. T. Eendebak, B. T. Buijtendorp, U. Mukhopadhyay, T. Fujita, C. Reichl, W. Wegscheider, L. M. K. Vandersypen

Appl. Phys. Lett. 113, 033101 (2018), (code: https://github.com/QuTech-Delft/qtt)

\section{Efficiently measuring a quantum device using machine learning} Authors: D. T. Lennon, H. Moon, L. C. Camenzind, Liuqi Yu, D. M. Zumbühl, G. A. D. Briggs, M. A. Osborne, E. A. Laird, N. Ares arXiv:1810.10042 (code: https://github.com/returnddd/CVAE_for_QE/)

\section{Recommended with a Commentary by Anton Akhmerov, Kavli Institute of Nanoscience, Delft University of Technology}

My optimism about applications of machine learning to condensed matter physics is very cautious. In an earlier commentary [1] I argued that condensed matter physics is unlike identifying cat photos, and therefore we might not benefit from going further than a robust linear regression. I believe the main reason for my argument still holds: because we deal with controlled problems and know a large part of the answer in advance, our data is often too simple to warrant machine learning. When writing this, I was however mostly considering applications of machine learning to theory.

Experiments are a different story, and they require a lot of work invisible to a theorist's eye. The largest hurdle in advanced experiments is not the observation of the relevant phenomenon, but bringing the device to a correct regime and calibrating it. I have once visited an optics lab, where the $\mathrm{Ph}$. D. students would spend every single morning measuring the parameter drift that occurred overnight and compensating for it. Then after lunch, an afternoon of measurements and a night of parameter drift, everything would repeat again. Likewise tuning a system of multiple quantum dots may take months of painstaking work regardless of whether the goal is to observe Kondo physics, make a spin qubit, or simulate a Hubbard model. Both works that I discuss tackle this problem using an automated approach.

In the work of van Diepen et al. the authors use something I would call a physicist's approach: their tuning protocol relies on a physics-based model of their device. The algorithm starts by identifying areas in the space of gate voltages that matches to different charge states. Then it fits all the observed anticrossings between energy levels to an effective model in order to determine couplings of different gates to individual levels and to define the so-called "virtual gates" that only control a single dot. At the last step, iterative tuning 
of the remaining parameters fixes the tunnel couplings between quantum dots to the desired value.

In contrast, the approach by Lennon et al. is machine learning all the way. The authors aim to characterize a single quantum dot in as few measurements as possible. They start from assembling a dataset of such measurements, augment it using simplified simulations, and also enrich it by applying additional transformations such as voltage rescaling. Using this training dataset they tune a neural network capable of describing any Coulomb blockage measurement with only a few parameters. Finally, when performing the measurement, they compute the distribution of these latent parameters and predict which new data points would result in a maximal information gain. As a result, they obtain all the relevant information about a device some 4 times faster.

The two approaches - a system-aware explicit tuning procedure and a completely generic neural network approach - correspond to two opposing opinions within the machine learning community. One part of the spectrum [2] argues that general purpose neural networks, while achieving impressive results, are lagging far behind humans in flexibility and quality of the trained models. While AlphaGo is impressive, they argue, it might become completely useless as soon as the rules of the game are even slightly changed. Paraphrasing in quantum dot terms, a neural network designed to analyze a single quantum dot may be completely useless when tackling double quantum dots, and one would need to retrain it from scratch even though it achieves good performance on a single dot. The opposing side [3] observes that the most impressive results in machine learning were so far achieved by relying on general purpose algorithms and the ever growing computational power rather than hard-coding the human knowledge and concepts into the algorithms. This argument suggests that as we produce more and more controlled systems, the effort going into describing all the minute physics details will eventually become unmanageable, and the only way forward would be to use a general purpose algorithm that relies on vast computational resources.

Automation seems to be the only way to reliably perform sophisticated measurements of fragile quantum devices. I cannot conclude which approach - a detailed physics-aware description, or an advanced neural network - will turn out to be more useful in the long run. It could even turn out that the optimal choice is a balance between the two approaches. We might need to learn how to build and train physics-aware neural networks capable of using human-defined concepts like Landau-Zener transitions or elastic cotunneling on the one hand and introduce their own models of the physical reality on the other.

\section{References}

[1] A. Akhmerov, "Machine learning versus human understanding", Journal Club for Condensed Matter Physics, May, 2017. Available at https://www. condmatjclub.org/?p= 3151.

[2] Brenden M. Lake, Tomer D. Ullman, Joshua B. Tenenbaum, Samuel J. Gershman arXiv:1604.00289 (2016).

[3] Richard Sutton "The Bitter Lesson" http://www.incompleteideas.net/IncIdeas/ BitterLesson.html (2019). 